\title{
A Transcriptomic study of probenecid on injured spinal cords in mice
}

\author{
Yu-Xin Zhang Equal first author, 1, 2, 3 , Sai-Nan Wang Equal first author, 1, 2 , Jing Chen ${ }^{1,2}$, Jian-Guo Hu ${ }^{\text {Corresp., 1, } 2}$, He-Zuo Lü ${ }^{\text {Corresp. 1, } 2}$ \\ ${ }^{1}$ Clinical Laboratory, the First Affiliated Hospital of Bengbu Medical College, Bengbu, China \\ 2 Anhui Key Laboratory of Tissue Transplantation, the First Affiliated Hospital of Bengbu Medical College, Bengbu, China \\ 3 Department of Biochemistry and Molecular Biology, Bengbu Medical College, Bengbu, China \\ Corresponding Authors: Jian-Guo Hu, He-Zuo Lü \\ Email address: jghu9200@163.com, Ihz233003@163.com
}

Background. Recent studies have found that probenecid has neuroprotective and reparative effects on central nervous system (CNS) injuries. However, its effect on genome-wide transcription in acute spinal cord injury $(\mathrm{SCl})$ remains unknown. In the present study, RNA sequencing (RNA-Seq) is used to analyze the effect of probenecid on the local expression of gene transcription eight hours after spinal injury. Methods. An Infinite Horizon impactor was used to perform contusive $\mathrm{SCl}$ in mice. The $\mathrm{SCl}$ model was made by using a rod ( $1.3 \mathrm{~mm}$ diameter) with a force of $50 \mathrm{Kdynes}$. Sham-operated mice only received a laminectomy without contusive injury. The injured mice were randomly assigned into either the control $\left(\mathrm{SCl}_{-} \mathrm{C}\right)$ or probenecid injection $(\mathrm{SCl} P$ ) group. In the latter group, the probenecid drug was intraperitoneally injected $(0.5 \mathrm{mg} / \mathrm{kg})$ immediately following injury. Eight hours after the injury or laminectomy, the spinal cords were removed from the mice in both groups. The total RNAs were extracted and purified for library preparation and transcriptome sequencing. Differential gene expressions (DEGs) of the three groups - sham, SCl_C and SCl_P - were analyzed using a DESeq software. Gene Ontology (GO) and Kyoto Encyclopedia of Genes and Genomes (KEGG) enrichment analysis of DEGs were performed using a GOseq R package and KOBAS software. Real-time quantitative reverse-transcriptase polymerase chain reaction (RT-qPCR) was used to validate RNA-Seq results. Results. RNA-Seq showed that, compared to the $\mathrm{SCl} C \mathrm{C}$ group, the number of DEGs was 641 in the SCI_P group (286 upregulated and 355 downregulated). According to GO analysis, DEGs were most enriched in extracellular matrix, collagen trimer, protein bounding and sequence specific DNA binding. KEGG analysis showed that the most enriched pathways included: cell adhesion molecules (CAMs), Leukocyte transendothelial migration, ECM-receptor interactions, PI3K-Akt signaling pathways, hematopoietic cell lineages, focal adhesions, the Rap1 signaling pathway, etc. The sequence data have been deposited into a Sequence Read Archive 
(https://www.ncbi.nlm.nih.gov/sra/PRJNA554464). 


\section{A Transcriptomic study of probenecid on injured} 2 spinal cords in mice

3

4 Yu-Xin Zhang ${ }^{1,2,3 \&}$, Sai-Nan Wang ${ }^{1,2 \&}$, Jing Chen ${ }^{1,2}$, Jian-Guo Hu${ }^{1,2 *}$, He-Zuo Lü ${ }^{1,2^{*}}$

$6{ }^{1}$ Clinical Laboratory, the First Affiliated Hospital of Bengbu Medical College, Anhui 233004, 7 P.R. China

$8{ }^{2}$ Anhui Key Laboratory of Tissue Transplantation, the First Affiliated Hospital of Bengbu

9 Medical College, Anhui 233004, P.R. China

$10{ }^{3}$ Department of Biochemistry and Molecular Biology, Bengbu Medical College, Anhui 233030,

11 P.R. China

12

\& These authors contributed equally to this work

14

* Co-corresponding authors:

Jian-Guo $\mathrm{Hu}$

17 Email address: jghu9200@163.com

18 He-Zuo Lü

Email address: 1hz233003@163.com

Please address correspondence to:

He-Zuo Lü, M.D. Ph.D., Professor

Anhui Key Laboratory of Tissue Transplantation

the First Affiliated Hospital of Bengbu Medical College

287 Chang Huai Road

Bengbu 233004, P.R. China

27

Tel: +86-552-3170692

E-mail: 1hz233003@163.com 
34

35

36

37

\section{Abstract}

Background. Recent studies have found that probenecid has neuroprotective and reparative effects on central nervous system (CNS) injuries. However, its effect on genome-wide transcription in acute spinal cord injury (SCI) remains unknown. In the present study, RNA sequencing (RNA-Seq) is used to analyze the effect of probenecid on the local expression of gene transcription eight hours after spinal injury.

Methods. An Infinite Horizon impactor was used to perform contusive SCI in mice. The SCI model was made by using a rod (1.3 mm diameter) with a force of 50 Kdynes. Sham-operated mice only received a laminectomy without contusive injury. The injured mice were randomly assigned into either the control (SCI_C) or probenecid injection (SCI_P) group. In the latter group, the probenecid drug was intraperitoneally injected $(0.5 \mathrm{mg} / \mathrm{kg})$ immediately following injury. Eight hours after the injury or laminectomy, the spinal cords were removed from the mice in both groups. The total RNAs were extracted and purified for library preparation and transcriptome sequencing. Differential gene expressions (DEGs) of the three groups — sham, SCI_C and SCI_P — were analyzed using a DESeq software. Gene Ontology (GO) and Kyoto Encyclopedia of Genes and Genomes (KEGG) enrichment analysis of DEGs were performed using a GOseq R package and KOBAS software. Real-time quantitative reverse-transcriptase polymerase chain reaction (RT-qPCR) was used to validate RNA-Seq results.

Results. RNA-Seq showed that, compared to the SCI_C group, the number of DEGs was 641 in the SCI_P group (286 upregulated and 355 downregulated). According to GO analysis, DEGs were most enriched in extracellular matrix, collagen trimer, protein bounding and sequence specific DNA binding. KEGG analysis showed that the most enriched pathways included: cell adhesion molecules (CAMs), Leukocyte transendothelial migration, ECM-receptor interactions, 
58 PI3K-Akt signaling pathways, hematopoietic cell lineages, focal adhesions, the Rap1 signaling

59 pathway, etc. The sequence data have been deposited into a Sequence Read Archive

60 (https://www.ncbi.nlm.nih.gov/sra/PRJNA554464).

61

\section{Introduction}

63 The term spinal cord injury (SCI) refers to a variety of injuries to the spinal cord. According to

64 the severity of injury the symptoms may vary, ranging from pain to a complete loss of movement

65 and sensory function. SCI affects millions of people worldwide, typically for life (Friedli et al.

66 2015). In the United States, there are around 12,000 to 20,000 new SCI cases a year, with more

67 than 280,000 patients confined to wheelchairs (Singh et al. 2014). In the past decade, the SCI

68 cases in China have risen tenfold, and are now increasing by about 60,000 cases every year (Qiu

69 2009). SCI has a high rate of disability and mortality, which creates a heavy burden for patients,

70 their families and society (Krueger et al. 2013). Therefore, it is imperative to explore effective

71 treatment methods for repairing SCI in order to improve the quality of life of patients and reduce

72 the burden of social medical care.

73 Pathological processes following traumatic SCI can be characterized as primary and secondary

74 injuries (Geisler et al. 2002; McDonald \& Sadowsky 2002). Primary injury refers to the direct

75 injury of the spinal cord by mechanical force, including compression, contusion, laceration and

76 penetration. Secondary injury refers to edema, ischemia, local inflammation and electrolyte

77 changes. These changes can cause an accumulation of lipid peroxides and oxygen-free radicals,

78 as well as a release of inflammatory factors and proteases. This can ultimately lead to a large

79 amount of cell apoptosis or necrosis, which further aggravates the damage to the neurons and

80 axons (Ahuja et al. 2017; Oyinbo 2011; Tran et al. 2018). 
81 Probenecid is an organic anion-transport protein inhibitor, which has been widely used in clinical

82 settings (Hagos et al. 2017; Tollner et al. 2015). For example, probenecid has been used as a

83 synergist in the treatment of gout and antibiotics (Baranova et al. 2004; Papadopoulos \&

84 Verkman 2008). It can reduce the degree of cognitive impairment in afflicted rats (Mawhinney et

85 al. 2011), as well as reverse cerebral ischemic injury and cellular inflammation (Wei et al. 2015;

86 Xiong et al. 2014). The combination of probenecid and N-Acetylcysteine could potentially both

87 maintain intracellular GSH concentrations and inhibit neuronal death after a traumatic stretch

88 injury (Du et al. 2016). Some studies report that probenecid can also reduce neuropathic pain in

89 the spinal cord (Bravo et al. 2014; Pineda-Farias et al. 2013). Therefore, these reports indicate

90 that probenecid has neuroprotective and reparative effects on central nervous system (CNS)

91 injuries. However, whether the drug can play a role in treating SCI and whether it can affect the

92 gene expression profiles in injured spinal cords remain unknown. To test this, probenecid was

93 injected intraperitoneally into spinal cord-injured mice immediately after injury. Eight hours

94 after the injury or laminectomy, the spinal cords were removed and RNA-Seq was used to

95 analyze the changes in transcriptome expression in the injured area. Then, the key molecules and

96 signal pathways were screened and identified, providing a new theoretical and experimental

97 basis for SCI clinical treatment.

\section{Materials \& Methods}

\section{Animals}

101 A total of 27 healthy, clean C57BL/6 female mice (18-20g, 8 weeks old) were used to model

102 SCI. The Animal Care and Use Committee of Bengbu Medical College provided full approval

103 for this research (037/2017). Animal care following surgery was carried out in compliance with 
104 the regulations for the management of experimental animals (revised by the Ministry of Science

105 and Technology of China in June 2004), as well as the guidelines and policies on rodent survival

106 surgery provided by the Animal Care and Use Committee of Bengbu Medical College.

107 Contusive SCI and drug injection

108 An Infinite Horizon impactor (Precision Systems \& Instrumentation, Lexington, KY) was used

109 to perform contusive SCI on the mice. These mice were first anesthetized with $50 \mathrm{mg} / \mathrm{kg}$

110 pentobarbital, followed by the excision of the T9 lamina. A SCI model of this procedure was

111 created using a rod (1.3 mm diameter) with a force of $50 \mathrm{Kdynes}$. Sham-operated (sham) mice

112 only received a laminectomy without contusive injury.

113 The spinal cord-injured mice were randomly assigned to the solvent control (SCI_C) or

114 probenecid injection (SCI_P) group. The solvent or probenecid $(0.5 \mathrm{mg} / \mathrm{kg})$ was intraperitoneally

115 injected immediately following injury. The solution ( $\mathrm{pH}$ 7.3) was prepared as previously

116 described (Hainz et al. 2017).

117 RNA isolation, quantification and qualification

118 Eight hours after the injury or laminectomy, the mice were anesthetized and perfused with $10 \mathrm{ml}$

119 PBS. Their spinal cords $(0.5 \mathrm{~cm}$ including the injury center $)$ were then removed. The total RNAs

120 from their spinal cords were extracted and purified as previously described (Shi et al. 2017).

121 Library preparation and transcriptome sequencing

122 The sequencing libraries were produced by using a NEBNext ${ }^{\circledR}$ Ultra $^{\mathrm{TM}}$ RNA Library Prep Kit for

123 Illumina $^{\circledR}$ (NEB, USA) as previously described (Shi et al. 2017). Finally, the $125 \mathrm{bp} / 150 \mathrm{bp}$

124 paired-end reads were obtained and sequenced on an Illumina Hiseq platform.

125 Analysis of differentially expressed gene (DEG) 
126 Prior to DEG analysis, the gene expression statistics were analyzed using RSEM software

127 (http://deweylab.github.io/RSEM/) to convert the read count numbers to Fragments Per Kilobase

128 of transcript per Million fragments mapped (FPKM), and Principal Component Analysis (PCA)

129 analysis was conducted to determine the similarities and differences in the data. DEGs of the

130 three groups of mice were analyzed as previously described (Shi et al. 2017) using DESeq

131 software (http://www.bioconductor.org/). Benjamini and Hochberg's approach was used to

132 control the false discovery rate and adjust the P-values. The adjusted P-value $<0.05$ was defined

133 as a standard for significant differences in gene expression. In addition to FPKM hierarchical

134 clustering analysis of DEGs, we further analyzed the subclusters based on $\log 2$ (ratios) of their

135 gene expression level relative to that of sham group. The $\log 2$ (ratios) in the SCI_C group $\geq 1$ or

$136 \leq-1$ was used as a cut-off for subcluster analysis. The clustering algorithm divided the DEGs

137 with similar gene expression trends into several subclusters.

138 Gene Ontology (GO) and Kyoto Encyclopedia of Genes and Genomes (KEGG) enrichment 139 analysis of DEGs

140 The GO and KEGG analysis were performed using a GOseq R package and KOBAS software as

141 previously described (Shi et al. 2017). In GO analysis, DEGs were implemented using the

142 GOseq R package and gene length bias was corrected. GO terms with corrected P value $\leq 0.05$

143 were considered significantly enriched by DEGs. KEGG is a database resource for understanding

144 the high-level functions and utilities of the biological system (http://www.genome.jp/kegg/). In

145 this study, we used KOBAS software to test the statistical enrichment of DEGs in KEGG

146 pathways.

147 Real-time quantitative reverse-transcriptase polymerase chain reaction (RT-qPCR) 
148 To validate RNA-Seq results, 9 DEGs were randomly selected and verified via RT-qPCR

149 according to our previous methods (Shi et al. 2017). The analysis was performed in 6 samples,

150 which included 3 independent samples and duplicates of these samples to be used in RNA-seq

151 analysis. PCR primer sequences are listed in Table 1. The relative quantitative results of each

152 group of genes were calculated according to the formula " $\Delta \Delta \mathrm{Ct}$ " (Livak \& Schmittgen 2001).

153 The statistical values ( $\mathrm{n}=6$ /group) were presented as mean \pm standard deviation (SD). The data

154 were analyzed using one-way Analysis of Variance (ANOVA), followed by Student-Newman-

155 Keuls tests. Statistical differences were considered significant at $\mathrm{P}<0.05$.

156

157 Results

\section{Identification of expressed transcripts the mice spinal cords}

159 For the high quality assessment of sequencing data, nine cDNA libraries were established, 160 including sham (sham_1, sham_2 and sham_3), SCI_C (SCI_C1, SCI_C2 and SCI_C3) and

161 SCI_P (SCI_P1, SCI_P2 and SCI_P3). RNA-Seq produced 48,848,744 - 61,037,096 raw reads

162 for each sample. After filtering out the low-quality reads, there were 48,226,002 - 60,037,772

163 clean reads, with the Q30 (\%) 93.67 - 94.31 (Table 2).

164 In order to identify the source of variation within the original data, PCA analysis was conducted.

165 As shown in Fig.1, PC1, PC2 and PC3 were 54.51, 12.33 and 7.09\%, respectively. Although not 166 too far from one another, the distance between SCI_C (or SCI_P) and sham was apparent and 167 sufficient for the analysis. These distances demonstrated that the data could be used for the next 168 analysis.

169 Effect of SCI and probenecid treatment on gene expression 170 
172 RPKM and DEGSeq were used to analyze the gene expression level and differential expression

173 profiles, respectively. The results showed that, as compared to the sham group, there were 4,617

174 DEGs in the SCI_C group, including 2,904 upregulated and 1,713 downregulated genes (Fig.2A

175 and Table S1). Compared to the SCI_C group, there were 641 different genes in the SCI_P

176 group, 286 were upregulated and 355 were downregulated (Fig.2B and Table S1). The sequence

177 data have been deposited into Sequence Read Archive

178 (https://www.ncbi.nlm.nih.gov/sra/PRJNA554464).

179 RT-qPCR identification of DEGs

180 In order to verify the RNA-Seq results, nine DEGs were randomly selected from the SCI_P

181 group, as compared with the SCI_C group, namely Itga1, Lamb1, Cldn5, Lama2, CD34, Esam,

182 Setdb2, Agrn and Ccnt2. The RNA-Seq and RT-qPCR results indicated that the expression

183 patterns of these DEGs were similar (Fig.3).

184 Cluster Analysis of DEGs

185 The DEGs from different groups were analyzed using FPKM hierarchical cluster analysis. As

186 shown in Fig. 4, DEGs were hierarchically clustered and classified into different expression

187 clusters. These clusters contained upregulated or downregulated DEGs. When compared to the

188 sham group, most upregulated DEGs in the SCI_C group were in the middle and upper clusters,

189 while downregulated DEGs were delegated to the lower cluster. Additionally, compared to the

190 sham group, most upregulated DEGs in the SCI_P group were in the upper cluster, while

191 downregulated DEGs were mainly observed in the lower cluster. When compared to the SCI_C

192 group, some upregulated DEGs in the SCI_P group were observed in upper cluster, while 
193 downregulated DEGs were observed in the middle cluster (there were also some clusters in this 194 grouping that showed no significant differences).

195 In addition to FPKM hierarchical clustering analysis of DEGs, the subclusters - which have 196 similar expression trends - were further analyzed. The $\log 2$ (ratios) in the SCI_C group $\geq 1$ or $\leq$ $197-1$ was used as a cut-off for subcluster analysis. As shown in Fig. 5, we found several subclusters 198 with similar expression trends. Based on $\log 2$ (ratios) of their gene expression levels relative to 199 that of the sham group, the $\log 2$ (ratios) of all gene expression levels in the sham group were 200 zero. Fig. $5 \mathrm{~A}$ and B show that the two subclusters were strongly upregulated following SCI and 201 then downregulated upon probenecid treatment. Fig. $5 \mathrm{C}$ and D show that the two subclusters 202 were strongly downregulated following SCI and then upregulated upon probenecid treatment. In 203 Fig. 5A, six genes (Cybb, Esam, Itgam, Itgb2, Msn and Ncf2) are demonstrated to have been 204 involved in the leukocyte transendothelial migration signaling pathway; six genes (Col4a1, 205 Erbb2, Flt4, Nos3, Syk and Thbs4) were also involved in the PI3K-Akt signaling pathway. Fig. 206 5B displays three genes (Cyba, Ncf1 and Rac2) involved in the NADPH oxidases, two genes 207 (Cflar and Tnfrsf10b) involved in the TRAIL signaling pathway and eight genes (Cd63, Cyba, 208 Ddx58, Fcer1g, Lyn, Myh9, Ncf1 and Psmb8) involved in the innate immune system. Fig. 5C 209 and D show that no gene can be clustered into valuable signaling pathways.

\section{GO enrichment analysis of DEGs}

211 When compared with the sham group, there were seventy-eight GO terms in upregulated DEGs

212 (Fig.6A, Table S2) and nine GO terms in downregulated DEGs (Fig.6B, Table S2) in the SCI_C 213 group. The upregulated DEGs were most enriched in: binding, protein binding, chemokine 214 activity, chemokine receptor binding, G-protein coupled receptor binding, anion binding, small 215 GTPase mediated signal transduction, immune system process, immune response. The 
216 downregulated DEGs were most enriched in: protein binding, binding, extracellular-glutamate217 gated ion channel activity, acid phosphatase activity, transporter activity, mannose metabolic 218 process, excitatory extracellular ligand-gated ion channel activity, transmembrane transporter 219 activity, anion transmembrane and transporter activity. In the SCI_P group, we observed three 220 GO terms in downregulated DEGs (Fig.6C, Table S3) and no valuable terms in upregulated 221 DEGs (Table S3) compared to the SCI_C group. The downregulated DEGs were protein 222 binding, binding and sequence-specific DNA binding.

\section{KEGG enrichment analysis of DEGs}

224 Scatter plots were used to express the KEGG enrichment analysis results for the DEGs. When 225 compared to the sham group, the upregulated DEGs in the SCI_C group were most enriched in

TNF, NF-kappa B, cytokine-cytokine receptor interaction, Toll-like receptor, Leukocyte transendothelial migration, PI3K-Akt, Focal adhesion and apoptosis. (Fig.7A, Table S4); the downregulated DEGs were most enriched in glutamatergic synapse, basal cell carcinoma, axon 229 guidance, other glycan degradation and nicotine addiction (Fig.7B, Table S4). In the SCI_P group vs. SCI_C group, only the "ECM-receptor interaction" was enriched in the upregulated DEGs (Fig.7C, Table S5); the downregulated DEGs were enriched in cell adhesion molecules (CAMs), malaria, leukocyte transendothelial migration, ECM-receptor interaction, PI3K-Akt signaling pathway, hematopoietic cell lineage, focal adhesion, Rap1 signaling pathway and amoebiasis (Fig.7D, Table S5).

235

236

\section{Discussion}

237 Recent studies have shown that probenecid has neuroprotective and repairing effects on brain 238 disorders (Wei et al. 2015; Xiong et al. 2014). However, its effect on genome-wide transcription 
239 in SCI is still unknown. Therefore, in this study, RNA-Seq was used to analyze the effect of

240 probenecid on the local expression of gene transcription eight hours after SCI. The results

241 showed that when compared with the sham group, there were 4,617 DEGs in the SCI_C group,

242 including 2,904 upregulated and 1,713 downregulated genes. When compared with the SCI_C

243 group, there were 641 DEGs in the SCI_P group, 286 of which were upregulated and 355

244 downregulated. These are consistent with others' and our previous reports (Chen et al. 2013; Shi

245 et al. 2017). It also shows that the results of this experiment are reliable. As compared to the

246 SCI_C, there were 641 DEGs in the SCI_P group, 286 were upregulated and 355 were

247 downregulated. To further verify the RNA-seq results, we randomly selected 9 DEGs (Itga1,

248 Lamb1, Cldn5, Lama2, CD34, Esam, Setdb2, Agrn and Ccnt2) for RT-qPCR. The results

249 showed that the expression patterns of these genes detected by these two methods were similar,

250 indicating that our RNA-seq results are reliable and can be used for subsequent analysis. These

251 also confirmed that probenecid can alter gene transcription after SCI.

252 To further analyze the DEGs effected by probenecid, we used GO enrichment to reflect the

253 distribution of DEGs on GO term enriched in cell components, molecular functions and

254 biological processes (Huang et al. 2013). In the SCI_P vs. SCI_C group, analysis showed that

255 there were $3 \mathrm{GO}$ terms in downregulated DEGs (protein binding, binding and sequence-specific

256 DNA binding) and no valuable terms in upregulated DEGs. KEGG analysis showed that the

257 valuable signaling pathways associated with these DEGs included: CAMs, leukocyte

258 transendothelial migration, ECM-receptor interaction, PI3K-Akt signaling pathway,

259 hematopoietic cell lineage, focal adhesion,-and Rap1 signaling pathway,ete.

260 Among these signal pathways, some are known to be related to SCI, such as CAMs (Brook et al.

261 2000; Zhang et al. 2008), ECM-receptor interaction (Zhou et al. 2017), PI3K-Akt signaling 
262 pathway (Li et al. 2019a; Li et al. 2019b; Zhang et al. 2017) and focal adhesion (Chuang et al. 263 2018; Graham et al. 2016; Hao et al. 2018).

264 Following SCI, probenecid treatment could downregulate some genes, subclusters and signaling 265 pathways. Leukocyte transendothelial migration from the blood into tissues is vital for immune 266 surveillance and inflammation (Cook-Mills 2006). However there is a large amount of leukocyte 267 infiltration involved in the pathological process of SCI. These infiltrating leukocytes need to bind 268 to endothelial cell adhesion molecules and then migrate between vascular endothelial cells 269 (Wang et al. 2011). Therefore, the inhibition of leukocyte transendothelial migration and CAMs 270 induced by probenecid may play a role in inhibiting inflammation by weakening the infiltration 271 of white blood cells in the injured area. In this study, we clustered six genes (Cybb, Esam, Itgam, 272 Itgb2, Msn and Ncf2) involved in this pathway. Their expression is strongly downregulated 273 following SCI and then upregulated upon probenecid treatment. This demonstrates that 274 probenecid treatment following SCI can play an anti-inflammatory role by inhibiting the 275 infiltration of inflammatory cells.

276 The ECM plays an important role in tissue and organ morphogenesis (Bonnans et al. 2014;

277 Rabelink et al. 2017) and control of cellular activities such as adhesion, migration, 278 differentiation, proliferation and apoptosis (Yue 2014). Focal adhesions are specialized, 279 intracellular sites in which aggregated integrin receptors interact with extracellular matrices, 280 while extracellular matrices interact with intracellular actin cytoskeleton (Burridge 2017; 281 LaFlamme et al. 2018). At the same time, focal adhesions are the result of cell and extracellular 282 matrix (ECM) interactions (Burridge 2017; De Pascalis \& Etienne-Manneville 2017). ECM and 283 focal adhesions are downregulated after probenecid treatment, indicating that probenecid might 284 improve SCI by inhibiting adhesion, migration, differentiation, proliferation and apoptosis. 
285 It has been reported that PI3K-Akt signaling fuses a variety of extracellular and intracellular 286 signal transduction pathways that regulate macrophage biology, including: the production of pro287 inflammatory cytokines, phagocytosis, autophagy and homeostasis (Vergadi et al. 2017). PI3K288 Akt signal pathway is downregulated in $\mathrm{SCI}$ after probenecid treatment, and the six genes 289 (Col4a1, Erbb2, Flt4, Nos3, Syk and Thbs4) being clustered into this pathway indicate that 290 probenecid might improve SCI by regulating macrophages and inhibiting inflammatory 291 pathways. This is likely to provide important clues towards identifying the mechanism by which 292 probenecid acts.

293 The relationship between the hematopoietic cell lineage pathway and SCI was referred to in a 294 report on the bioinformatics analysis of SCI (Zhu et al. 2017). Its specific role has yet to be 295 reported and requires further discussion.

296 Rap1 signal pathway plays an important role in regulating cell-cell and cell-matrix interactions 297 by regulating the function of adhesion molecules (Kim et al. 2011; Pollan et al. 2018). In our 298 study, Rap1 signaling pathways were enriched in downregulated DEGs of SCI after probenecid 299 treatment, suggesting that probenecid may inhibit cell adhesion and polarization by inhibiting the 300 Rap1 signaling pathway, thereby inhibiting inflammation.

301 In addition, three genes (Cyba, Ncf1 and Rac2) related to the NADPH oxidases, two genes (Cflar 302 and Tnfrsf10b) related to the TRAIL signaling pathway and eight genes (Cd63, Cyba, Ddx58, 303 Fcer1g, Lyn, Myh9, Ncf1 and Psmb8) related to the innate immune system were also strongly 304 downregulated following probenecid treatment. We know that NADPH oxidases are involved in 305 oxidative stress, the TRALL signaling pathway mediates inflammation and apoptosis and the 306 immune system is involved in almost all pathological processes of injury (Chyuan et al. 2018;

307 Ewald 2018; Tisato et al. 2018). Therefore, probenecid treatment can potentially play a 
308 neuroprotective role by inhibiting immune response, oxidative stress, anti-inflammation and anti-

309 apoptosis after SCI.

\section{Conclusions}

311 Acute SCI can lead to changes in the mRNAs of injured spinal cords. These mRNAs and their

312 related pathways can provide some explanation for the pathological mechanism of acute SCI.

313 More interestingly, we also demonstrated that probenecid can lead to gene expression inhibitions

314 in an acute injured spinal cord. These downregulated DEGs and their associated signaling

315 pathways — such as focal adhesions, leukocyte transendothelial migration, ECM-receptor

316 interaction, PI3K-Akt, Rap1 — are mainly related to inflammatory response, local hypoxia,

317 macrophage differentiation, adhesion migration and apoptosis of local cells. This suggests that

318 the application of probenecid in the acute phase can improve the local microenvironment of SCI.

319 However, the application of probenecid as a therapeutic drug for SCI requires further

320 investigation. Next, the detailed research on this subject will be conducted by combining animal

321 models and clinical practice.

322 
323

324

325

326

327

328

329

330

331

332

333

334

335

336

337

338

339

340

341

342

343

344

345

346

347

348

349

350

351

352

353

354

355

356

357

358

359

360

361

362

363

364

365

366

367

368

\section{References}

Ahuja CS, Nori S, Tetreault L, Wilson J, Kwon B, Harrop J, Choi D, and Fehlings MG. 2017. Traumatic Spinal Cord Injury-Repair and Regeneration. Neurosurgery 80:S9-S22. 10.1093/neuros/nyw080

Baranova A, Ivanov D, Petrash N, Pestova A, Skoblov M, Kelmanson I, Shagin D, Nazarenko S, Geraymovych E, Litvin O, Tiunova A, Born TL, Usman N, Staroverov D, Lukyanov S, and Panchin Y. 2004. The mammalian pannexin family is homologous to the invertebrate innexin gap junction proteins. Genomics 83:706-716. 10.1016/j.ygeno.2003.09.025

Bonnans C, Chou J, and Werb Z. 2014. Remodelling the extracellular matrix in development and disease. Nat Rev Mol Cell Biol 15:786-801. 10.1038/nrm3904

Bravo D, Ibarra P, Retamal J, Pelissier T, Laurido C, Hernandez A, and Constandil L. 2014. Pannexin 1: a novel participant in neuropathic pain signaling in the rat spinal cord. Pain 155:2108-2115. 10.1016/j.pain.2014.07.024

Brook GA, Houweling DA, Gieling RG, Hermanns T, Joosten EA, Bar DP, Gispen WH, Schmitt AB, Leprince P, Noth J, and Nacimiento W. 2000. Attempted endogenous tissue repair following experimental spinal cord injury in the rat: involvement of cell adhesion molecules L1 and NCAM? Eur J Neurosci 12:3224-3238.

Burridge K. 2017. Focal adhesions: a personal perspective on a half century of progress. FEBS $J$ 284:3355-3361. 10.1111/febs. 14195

Chen K, Deng S, Lu H, Zheng Y, Yang G, Kim D, Cao Q, and Wu JQ. 2013. RNA-seq characterization of spinal cord injury transcriptome in acute/subacute phases: a resource for understanding the pathology at the systems level. PLoS One 8:e72567. 10.1371/journal.pone.0072567

Chuang YC, Lee CH, Sun WH, and Chen CC. 2018. Involvement of advillin in somatosensory neuron subtype-specific axon regeneration and neuropathic pain. Proc Natl Acad Sci US A 115:E8557-E8566. 10.1073/pnas.1716470115

Chyuan IT, Tsai HF, Wu CS, Sung CC, and Hsu PN. 2018. TRAIL-Mediated Suppression of T Cell Receptor Signaling Inhibits T Cell Activation and Inflammation in Experimental Autoimmune Encephalomyelitis. Front Immunol 9:15. 10.3389/fimmu.2018.00015

Cook-Mills JM. 2006. Hydrogen peroxide activation of endothelial cell-associated MMPs during VCAM-1-dependent leukocyte migration. Cell Mol Biol (Noisy-le-grand) 52:8-16.

De Pascalis C, and Etienne-Manneville S. 2017. Single and collective cell migration: the mechanics of adhesions. Mol Biol Cell 28:1833-1846. 10.1091/mbc.E17-03-0134

Du L, Empey PE, Ji J, Chao H, Kochanek PM, Bayir H, and Clark RS. 2016. Probenecid and NAcetylcysteine Prevent Loss of Intracellular Glutathione and Inhibit Neuronal Death after Mechanical Stretch Injury In Vitro. J Neurotrauma 33:1913-1917. 10.1089/neu.2015.4342

Ewald CY. 2018. Redox Signaling of NADPH Oxidases Regulates Oxidative Stress Responses, Immunity and Aging. Antioxidants (Basel) 7. 10.3390/antiox7100130

Friedli L, Rosenzweig ES, Barraud Q, Schubert M, Dominici N, Awai L, Nielson JL, Musienko P, Nout-Lomas Y, Zhong H, Zdunowski S, Roy RR, Strand SC, van den Brand R, Havton LA, Beattie MS, Bresnahan JC, Bezard E, Bloch J, Edgerton VR, Ferguson AR, Curt A, Tuszynski MH, and Courtine G. 2015. Pronounced species divergence in corticospinal tract reorganization and functional recovery after lateralized spinal cord injury favors primates. Sci Transl Med 7:302ra134. 10.1126/scitranslmed.aac5811

Geisler FH, Coleman WP, Benzel E, Ducker T, and Hurlbert RJ. 2002. Spinal cord injury. Lancet 360:1883; author reply 1884. 10.1016/s0140-6736(02)11744-2 
369

370

371

372

373

374

375

376

377

378

379

380

381

382

383

384

385

386

387

388

389

390

391

392

393

394

395

396

397

398

399

400

401

402

403

404

405

406

407

408

409

410

411

412

413

414

Graham ZA, Qin W, Harlow LC, Ross NH, Bauman WA, Gallagher PM, and Cardozo CP. 2016. Focal adhesion kinase signaling is decreased 56 days following spinal cord injury in rat gastrocnemius. Spinal Cord 54:502-509. 10.1038/sc.2015.183

Hagos FT, Daood MJ, Ocque JA, Nolin TD, Bayir H, Poloyac SM, Kochanek PM, Clark RS, and Empey PE. 2017. Probenecid, an organic anion transporter 1 and 3 inhibitor, increases plasma and brain exposure of N-acetylcysteine. Xenobiotica 47:346-353. 10.1080/00498254.2016.1187777

Hainz N, Wolf S, Beck A, Wagenpfeil S, Tschernig T, and Meier C. 2017. Probenecid arrests the progression of pronounced clinical symptoms in a mouse model of multiple sclerosis. Sci Rep 7:17214. 10.1038/s41598-017-17517-5

Hao M, Ji XR, Chen H, Zhang W, Zhang LC, Zhang LH, Tang PF, and Lu N. 2018. Cell cycle and complement inhibitors may be specific for treatment of spinal cord injury in aged and young mice: Transcriptomic analyses. Neural Regen Res 13:518-527. 10.4103/16735374.226405

Huang Q, Wu LY, Wang Y, and Zhang XS. 2013. GOMA: functional enrichment analysis tool based on GO modules. Chin J Cancer 32:195-204. 10.5732/cjc.012.10151

Kim C, Ye F, and Ginsberg MH. 2011. Regulation of integrin activation. Annu Rev Cell Dev Biol 27:321-345. 10.1146/annurev-cellbio-100109-104104

Krueger H, Noonan VK, Trenaman LM, Joshi P, and Rivers CS. 2013. The economic burden of traumatic spinal cord injury in Canada. Chronic Dis Inj Can 33:113-122.

LaFlamme SE, Mathew-Steiner S, Singh N, Colello-Borges D, and Nieves B. 2018. Integrin and microtubule crosstalk in the regulation of cellular processes. Cell Mol Life Sci 75:41774185. 10.1007/s00018-018-2913-X

Li H, Zhang X, Qi X, Zhu X, and Cheng L. 2019a. Icariin Inhibits Endoplasmic Reticulum Stressinduced Neuronal Apoptosis after Spinal Cord Injury through Modulating the PI3K/AKT Signaling Pathway. Int J Biol Sci 15:277-286. 10.7150/ijbs.30348

Li Y, Guo Y, Fan Y, Tian H, Li K, and Mei X. 2019b. Melatonin Enhances Autophagy and Reduces Apoptosis to Promote Locomotor Recovery in Spinal Cord Injury via the PI3K/AKT/mTOR Signaling Pathway. Neurochem Res 44:2007-2019. 10.1007/s11064019-02838-W

Livak KJ, and Schmittgen TD. 2001. Analysis of relative gene expression data using real-time quantitative PCR and the 2(-Delta Delta C(T)) Method. Methods 25:402-408. 10.1006/meth.2001.1262

Mawhinney LJ, de Rivero Vaccari JP, Dale GA, Keane RW, and Bramlett HM. 2011. Heightened inflammasome activation is linked to age-related cognitive impairment in Fischer 344 rats. BMC Neurosci 12:123. 10.1186/1471-2202-12-123

McDonald JW, and Sadowsky C. 2002. Spinal-cord injury. Lancet 359:417-425. 10.1016/S01406736(02)07603-1

Oyinbo CA. 2011. Secondary injury mechanisms in traumatic spinal cord injury: a nugget of this multiply cascade. Acta Neurobiol Exp (Wars) 71:281-299.

Papadopoulos MC, and Verkman AS. 2008. Potential utility of aquaporin modulators for therapy of brain disorders. Prog Brain Res 170:589-601. 10.1016/S0079-6123(08)00446-9

Pineda-Farias JB, Perez-Severiano F, Gonzalez-Esquivel DF, Barragan-Iglesias P, BravoHernandez M, Cervantes-Duran C, Aguilera P, Rios C, and Granados-Soto V. 2013. The L-kynurenine-probenecid combination reduces neuropathic pain in rats. Eur J Pain 17:1365-1373. 10.1002/j.1532-2149.2013.00305.x 
415 Pollan SG, Huang F, Sperger JM, Lang JM, Morrissey C, Cress AE, Chu CY, Bhowmick NA, You

416

417

418

419

420

421

422

423

424

425

426

427

428

429

430

431

432

433

434

435

436

437

438

439

440

441

442

443

444

445

446

447

448

449

450

451

452

453

454

455

456

457

458

459

S, Freeman MR, Spassov DS, Moasser MM, Carter WG, Satapathy SR, Shah K, and Knudsen BS. 2018. Regulation of inside-out betal-integrin activation by CDCP1. Oncogene 37:2817-2836. 10.1038/s41388-018-0142-2

Qiu J. 2009. China Spinal Cord Injury Network: changes from within. Lancet Neurol 8:606-607. 10.1016/S1474-4422(09)70162-0

Rabelink TJ, van den Berg BM, Garsen M, Wang G, Elkin M, and van der Vlag J. 2017. Heparanase: roles in cell survival, extracellular matrix remodelling and the development of kidney disease. Nat Rev Nephrol 13:201-212. 10.1038/nrneph.2017.6

Shi LL, Zhang N, Xie XM, Chen YJ, Wang R, Shen L, Zhou JS, Hu JG, and Lu HZ. 2017. Transcriptome profile of rat genes in injured spinal cord at different stages by RNAsequencing. BMC Genomics 18:173. 10.1186/s12864-017-3532-x

Singh A, Tetreault L, Kalsi-Ryan S, Nouri A, and Fehlings MG. 2014. Global prevalence and incidence of traumatic spinal cord injury. Clin Epidemiol 6:309-331. 10.2147/CLEP.S68889

Tisato V, Gallo S, Melloni E, Celeghini C, Passaro A, Zauli G, Secchiero P, Bergamini C, Trentini A, Bonaccorsi G, Valacchi G, Zuliani G, and Cervellati C. 2018. TRAIL and Ceruloplasmin Inverse Correlation as a Representative Crosstalk between Inflammation and Oxidative Stress. Mediators Inflamm 2018:9629537. 10.1155/2018/9629537

Tollner K, Brandt C, Romermann K, and Loscher W. 2015. The organic anion transport inhibitor probenecid increases brain concentrations of the NKCC1 inhibitor bumetanide. Eur $J$ Pharmacol 746:167-173. 10.1016/j.ejphar.2014.11.019

Tran AP, Warren PM, and Silver J. 2018. The Biology of Regeneration Failure and Success After Spinal Cord Injury. Physiol Rev 98:881-917. 10.1152/physrev.00017.2017

Vergadi E, Ieronymaki E, Lyroni K, Vaporidi K, and Tsatsanis C. 2017. Akt Signaling Pathway in Macrophage Activation and M1/M2 Polarization. J Immunol 198:1006-1014. 10.4049/jimmunol.1601515

Wang JG, Williams JC, Davis BK, Jacobson K, Doerschuk CM, Ting JP, and Mackman N. 2011. Monocytic microparticles activate endothelial cells in an IL-1beta-dependent manner. Blood 118:2366-2374. 10.1182/blood-2011-01-330878

Wei R, Wang J, Xu Y, Yin B, He F, Du Y, Peng G, and Luo B. 2015. Probenecid protects against cerebral ischemia/reperfusion injury by inhibiting lysosomal and inflammatory damage in rats. Neuroscience 301:168-177. 10.1016/j.neuroscience.2015.05.070

Xiong XX, Gu LJ, Shen J, Kang XH, Zheng YY, Yue SB, and Zhu SM. 2014. Probenecid protects against transient focal cerebral ischemic injury by inhibiting HMGB1 release and attenuating AQP4 expression in mice. Neurochem Res 39:216-224. 10.1007/s11064-013$1212-\mathrm{z}$

Yue B. 2014. Biology of the extracellular matrix: an overview. J Glaucoma 23:S20-23. 10.1097/IJG.0000000000000108

Zhang F, Ru N, Shang ZH, Chen JF, Yan C, Li Y, and Liang J. 2017. Daidzein ameliorates spinal cord ischemia/reperfusion injury-induced neurological function deficits in SpragueDawley rats through PI3K/Akt signaling pathway. Exp Ther Med 14:4878-4886. 10.3892/etm.2017.5166

Zhang Y, Yeh J, Richardson PM, and Bo X. 2008. Cell adhesion molecules of the immunoglobulin superfamily in axonal regeneration and neural repair. Restor Neurol Neurosci 26:81-96.

Peer] reviewing PDF | (2019:07:39856:2:0:NEW 25 Nov 2019) 
460

461

462

463

464

465

466

467

468

469

470

471

472

473

474

475

476

477

478

479

480

481

482

483

484

485

486

487

488

489

490

491

492

493

494

495

496

497

498

499

500

501

502

503

504

Zhou J, Xiong Q, Chen H, Yang C, and Fan Y. 2017. Identification of the Spinal Expression Profile of Non-coding RNAs Involved in Neuropathic Pain Following Spared Nerve Injury by Sequence Analysis. Front Mol Neurosci 10:91. 10.3389/fnmol.2017.00091Zhu Z, Wang D, Jiao W, Chen G, Cao Y, Zhang Q, and Wang J. 2017. Bioinformatics analyses of pathways and gene predictions in IL-1alpha and IL-1beta knockout mice with spinal cord injury. Acta Histochem 119:663-670. 10.1016/j.acthis.2017.07.007

\section{Figures}

\section{Figure 1 PCA analysis}

PCA analysis was performed using three principal components (PC1, 2 and 3$)$ to demonstrate the source of variance $(n=3)$.

\section{Figure 2 Volcano map of DEGs}

Red, green and blue dots represent significantly upregulated, downregulated and unchanged gene expressions, respectively. (A) SCI_C vs Sham; (B) SCI_P vs SCI_C.

\section{Figure 3 RT-qPCR verification of DEGs characterized by RNA-Seq}

A: The longitudinal coordinates in RNA-Seq are mRNA expression levels (read counts, $n=3$ ). B: The longitudinal coordinates in RT-qPCR are mRNA expression level calculated using the " $\Delta \Delta \mathrm{Ct}$ " method and expressed relative to the value in the sham group (designated as 1). All data were calculated with mean \pm standard deviation $(n=6$, which included 3 independent samples and the 3 same samples used for the RNA-seq analysis). $* * \mathrm{P}<0.01$ (ANOVA).

\section{Figure 4 Hierarchical cluster analysis of DEGs}

The DEGs in different groups were analyzed using FPKM hierarchical cluster analysis. The read count numbers of FPKM were converted using a RSEM software. DEGs were classified into different expression cluster by hierarchical clustering. The colour scheme (red to blue) represents the up-to-down of the gene expression. sham: sham group; SCI_C: SCI (solvent control) group; SCI_P: SCI (probenecid) group.

\section{Figure 5 Subcluster analysis of DEGs}

The subclusters of DEGs which have similar expression trends were further analyzed. The $\log 2$ (ratios) in SCI_C group $\geq 1$ or $\leq-1$ was used as a cut-off. Based on log2 (ratios) of the gene expression level relative to that of sham group, the $\log 2$ (ratios) of all gene expression levels in sham group were zero. A and B: the two subclusters which were strongly upregulated following SCI and then downregulated upon probenecid treatment. C and D: the two subclusters which were strongly downregulated following SCI and then upregulated upon probenecid treatment.

\section{Figure 6 GO enrichment analysis of DEGs}


505 DEGs were implemented by the GOseq R package, in which gene length bias was corrected. GO 506 terms with corrected $P$ value $\leq 0.05$ were considered significantly enriched by DEGs. The asterisk

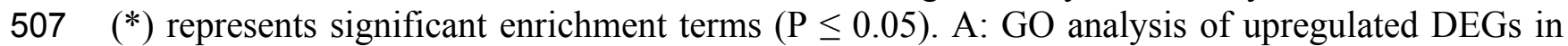
508 SCI_C vs sham group; B: GO analysis of downregulated DEGs in SCI_C vs sham group; C: GO 509 analysis of downregulated DEGs in SCI_P vs SCI_C group.

510

511

512

513

514

515

516

517

518

519

520

521

522

523

\section{Figure 7 KEGG enrichment analyses of DEGs}

KOBAS software was used to test the statistical enrichment of DEGs in KEGG pathways. In this figure, KEGG enrichment is measured by Rich factor, Qvalue and the number of genes enriched in the related pathway. Rich factor refers to the ratio of the number of differentiated genes (sample number) enriched in the pathway to the number of annotated genes (background number). The larger the Rich factor, the greater the degree of enrichment. Qvalue is the Pvalue after multiple hypothesis test correction. The range of Qvalue is between 0 and 1 . The closer the Qvalue is to 0, the more significant the enrichment is. The KEGG pathways were shown in A: upregulated DEGs (SCI_C vs sham); B: downregulated DEGs; C: upregulated DEGs (SCI_P vs SCP_C); D: downregulated DEGs (SCI_C vs sham).

\section{Tables}

524

525

526

527

528

529

Table 1 PCR primers used in the study

Table 2 Summary of sequence assembly after Illumina sequencing

\section{Supplementary materials}

530

531

Table S1 DEGs of different groups

Table S2 GO enrichment analysis of SCI_C vs sham group

532

Table S3 GO enrichment analysis of SCI_P vs SCI_C group

533

534

Table S4 KEGG analysis of SCI_C vs sham group

Table S5 KEGG analysis of SCI_P vs SCI_C group

535 
Figure 1

Figure 1 PCA analysis

PCA analysis was performed using three principal components ( $\mathrm{PC} 1,2$, and 3 ) to demonstrate the source of variance $(n=3)$.

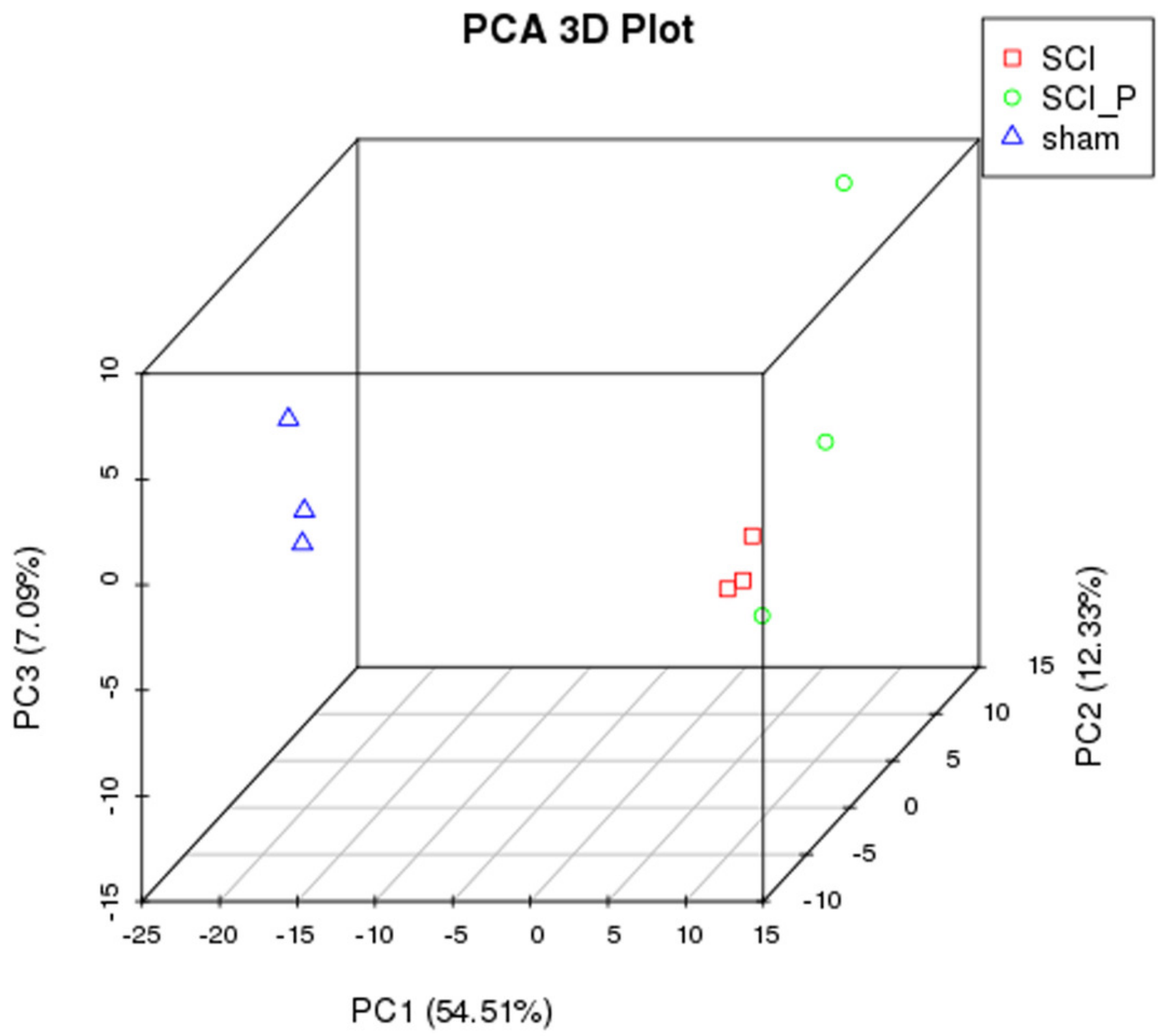


Figure 2

Figure 2 Volcano map of DEGs

Red, green and blue dots represent significantly upregulated, downregulated and no changed gene expressions, respectively. (A) $\mathrm{SCl} C \mathrm{C}$ vs Sham; (B) SCl_P vs $\mathrm{SCl} C$.

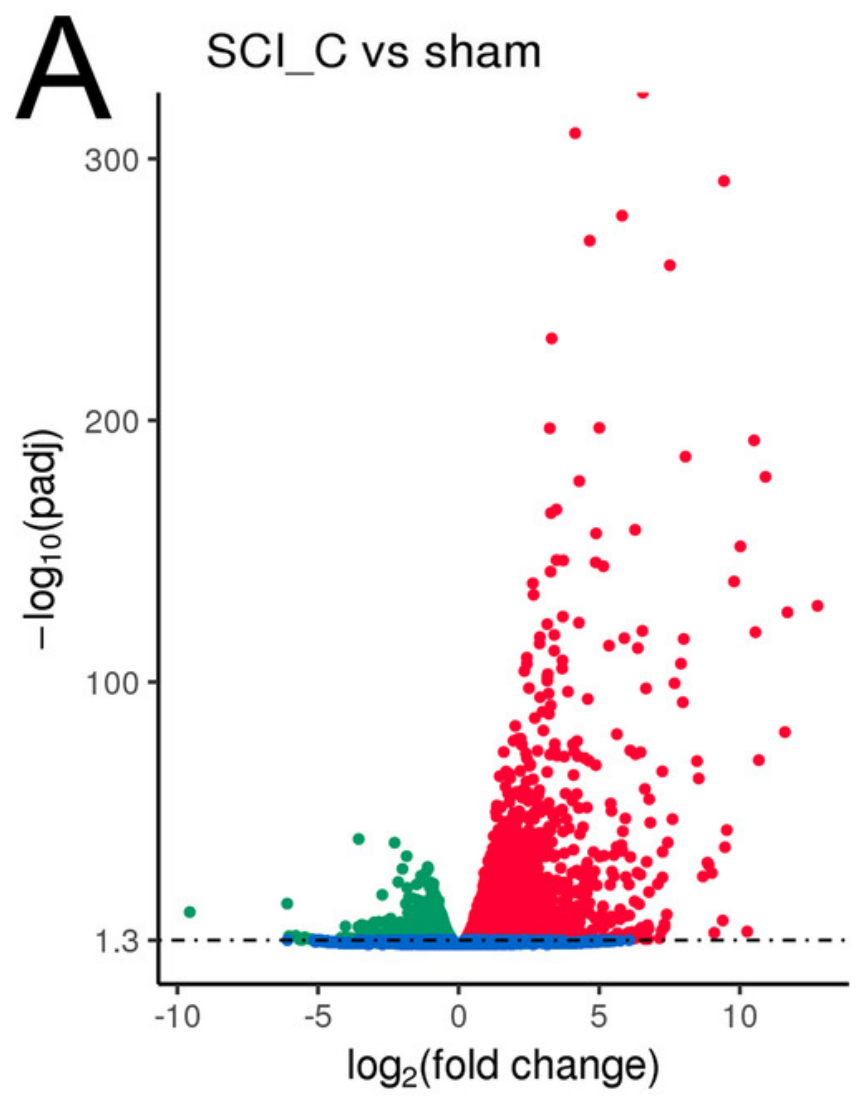

Differential Expressed Genes ( 4617 )

- up regulated: 2904

- down regulated: 1713

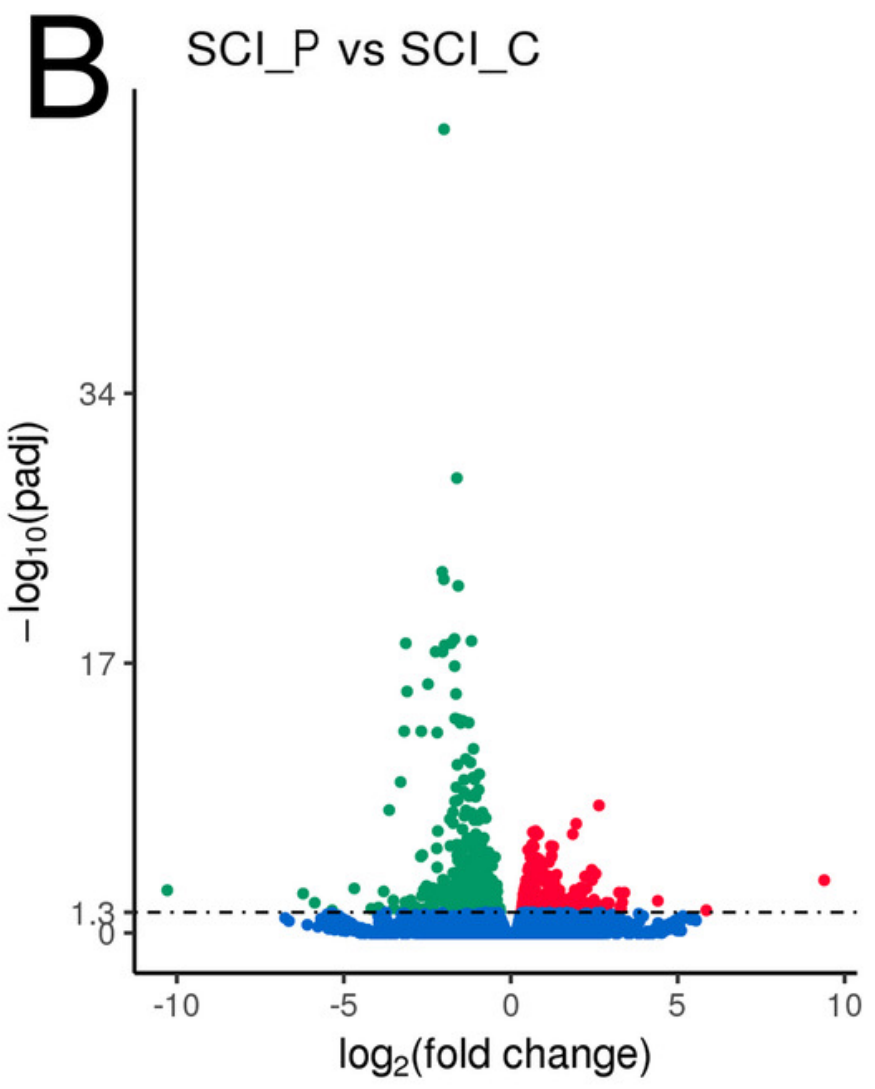

Differential Expressed Genes ( 641 )

- up regulated: 286

- down regulated: 355 


\section{Figure 3}

Figure 3 RT-qPCR verification of DEGs characterized by RNA-Seq

A: The longitudinal coordinates in RNA-Seq were the mRNA expression level (read counts, $n$ $=3$ ). $\mathrm{B}$ : The longitudinal coordinates in RT-qPCR were the mRNA expression level calculated using the ${ }^{\Delta \Lambda} \mathrm{Ct}$ method and expressed relative to the value in the sham group (designated as 1). All data were calculated with mean \pm standard deviation $(n=6$, which included 3 independent samples and the 3 same samples used for the RNA-seq analysis). ${ }^{* * P}<0.01$ (ANOVA). 

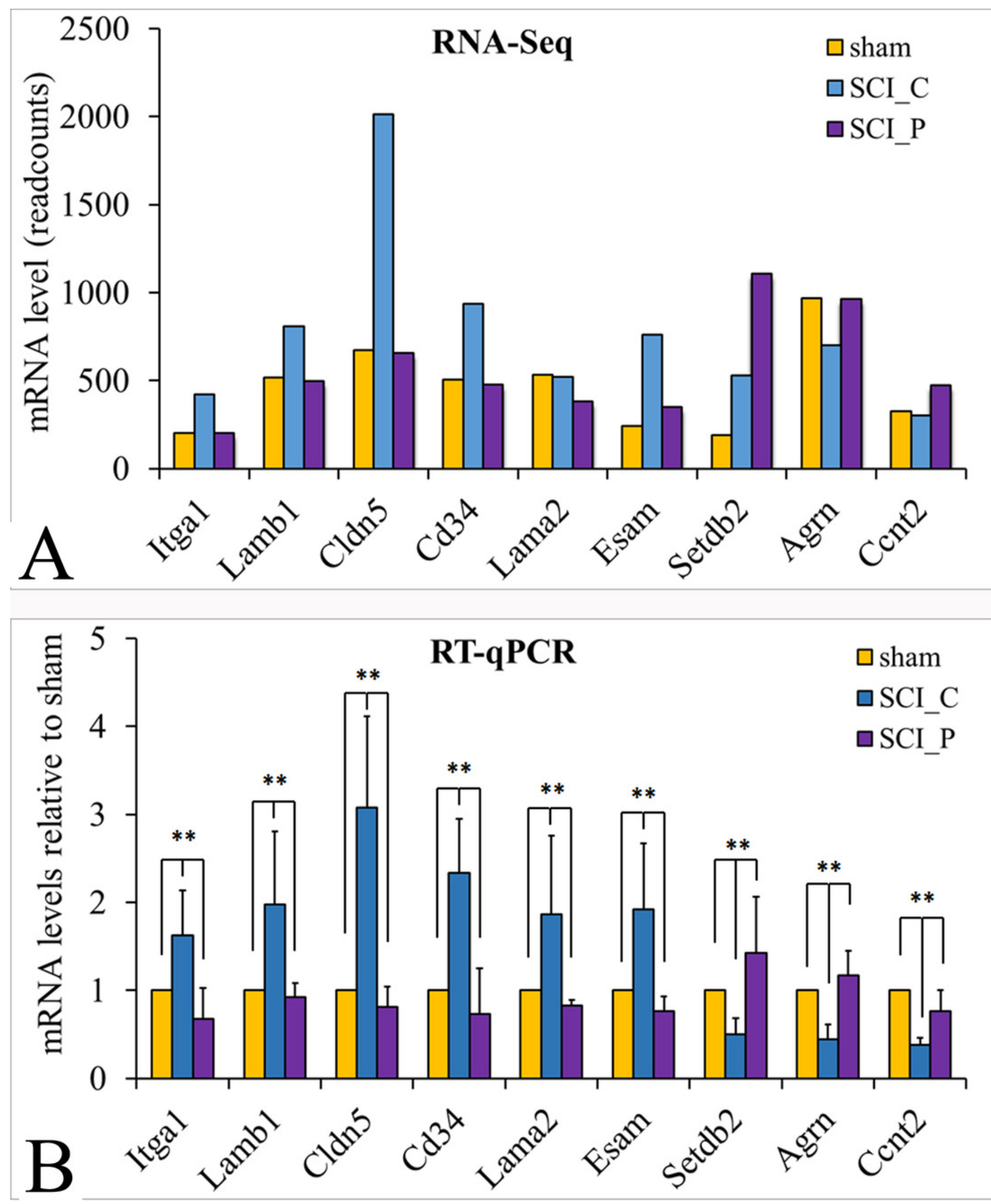


\section{Figure 4}

Figure 4 Hierarchical cluster analysis of DEGs

The DEGs in different groups were analyzed using FPKM hierarchical cluster analysis. The read count numbers of FPKM were converted by RSEM software. DEGs were classified into different expression cluster by hierarchical clustering. The colour scheme (red to blue) represents the up to down of the gene expression. sham: sham group; $\mathrm{SCl} \mathrm{C}_{-} \mathrm{SCl}$ (solvent control) group; SCl_P: SCl (probenecid) group. 


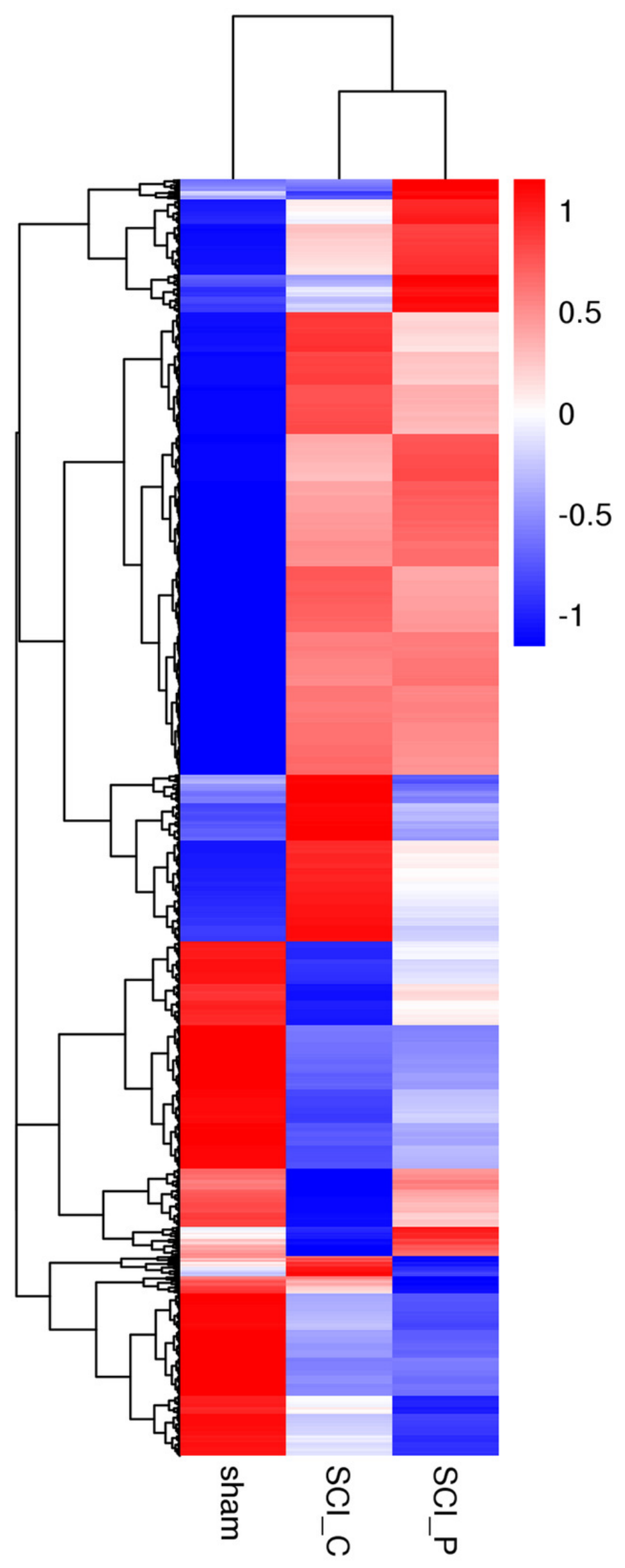




\section{Figure 5}

Figure 5 Subcluster analysis of DEGs

The subclusters of DEGs which have similar expression trends were further analyzed. The $\log 2$ (ratios) in $\mathrm{SCl}$ C group $\geq 1$ or $\leq-1$ was used as a cut-off. Based on log2 (ratios) of the gene expression level relative to that of sham group, the log2 (ratios) of all gene expression levels in sham group were zero. A and B: the two subclusters which were strongly upregulated following $\mathrm{SCl}$, and then downregulated upon probenecid treatment. $\mathrm{C}$ and $\mathrm{D}$ : the two subclusters which were strongly downregulated following $\mathrm{SCl}$, and then upregulated upon probenecid treatment. 


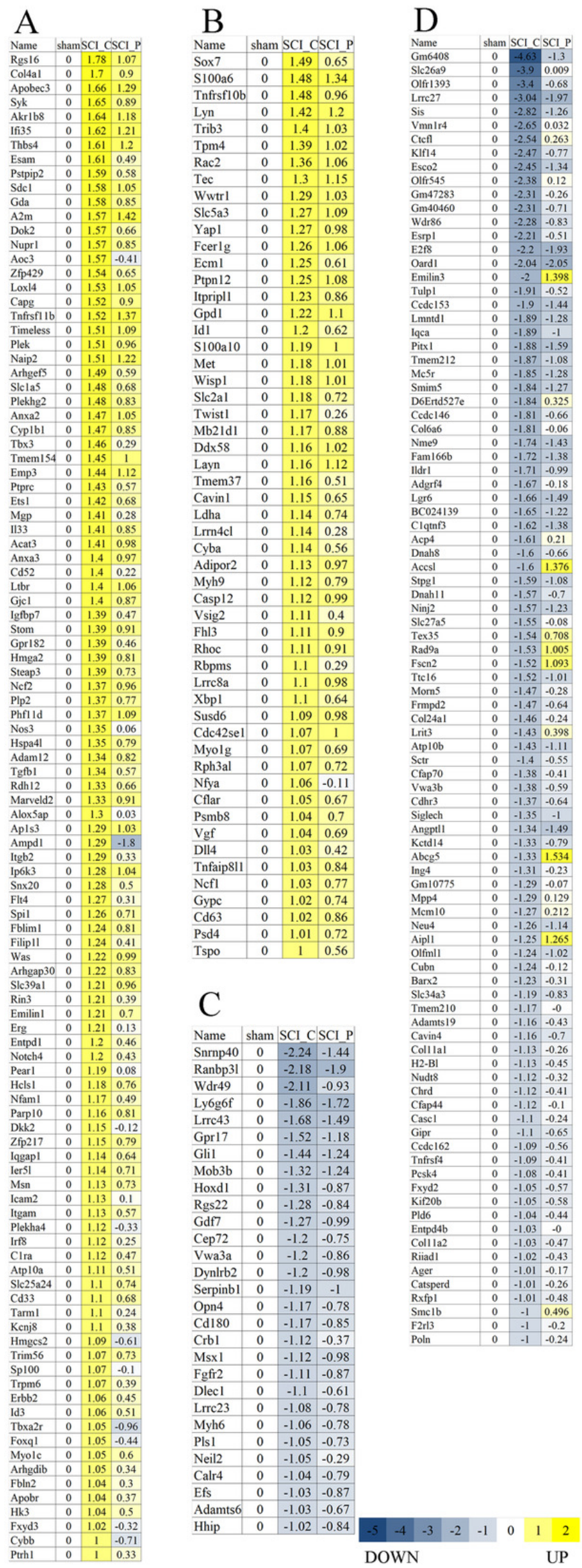




\section{Figure 6}

Figure $6 \mathrm{GO}$ enrichment analysis of DEGs

DEGs were implemented by the GOseq R package, in which gene length bias was corrected. $\mathrm{GO}$ terms with corrected $\mathrm{P}$ value $\leq 0.05$ were considered significantly enriched by DEGs. The asterisk $(*)$ represent significant enrichment terms $(P \leq 0.05)$. A: GO analysis of upregulated DEGs in SCl_C vs sham group; B: GO analysis of downregulated DEGs in $\mathrm{SCl} C \mathrm{C}$ vs sham group; $\mathrm{C}$ : $\mathrm{GO}$ analysis of downregulated $\mathrm{DEGs}$ in $\mathrm{SCl}$ _P vs $\mathrm{SCl}_{-} \mathrm{C}$ group. 
A

Enriched GO Terms (SCI_C vs sham) UP

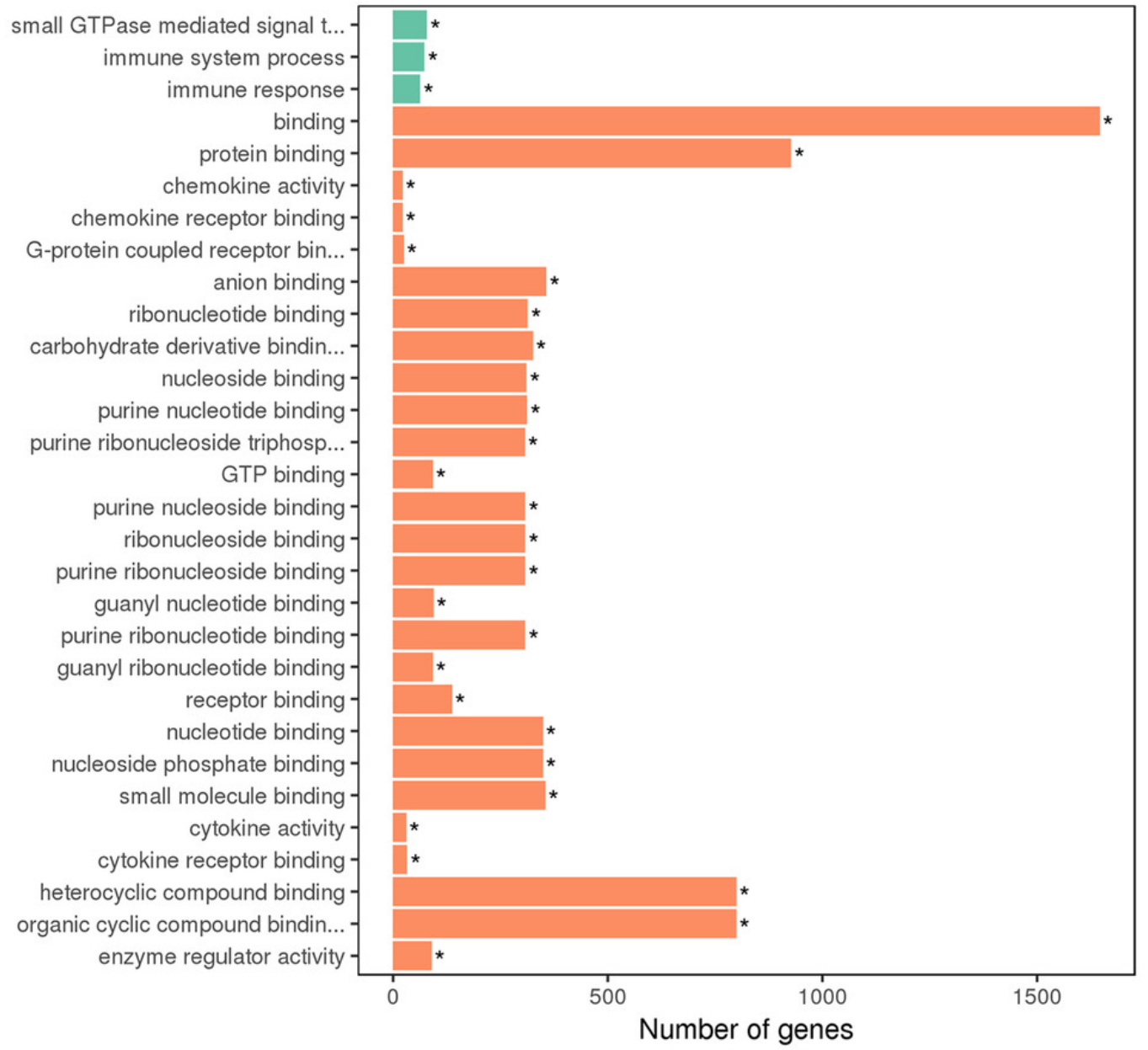

B

Enriched GO Terms (SCI_C vs sham) DOWN

mannose metabolic process protein binding binding

extracellular-glutamate-gated ... acid phosphatase activity transporter activity

excitatory extracellular ligan... transmembrane transporter acti... anion transmembrane transporte...
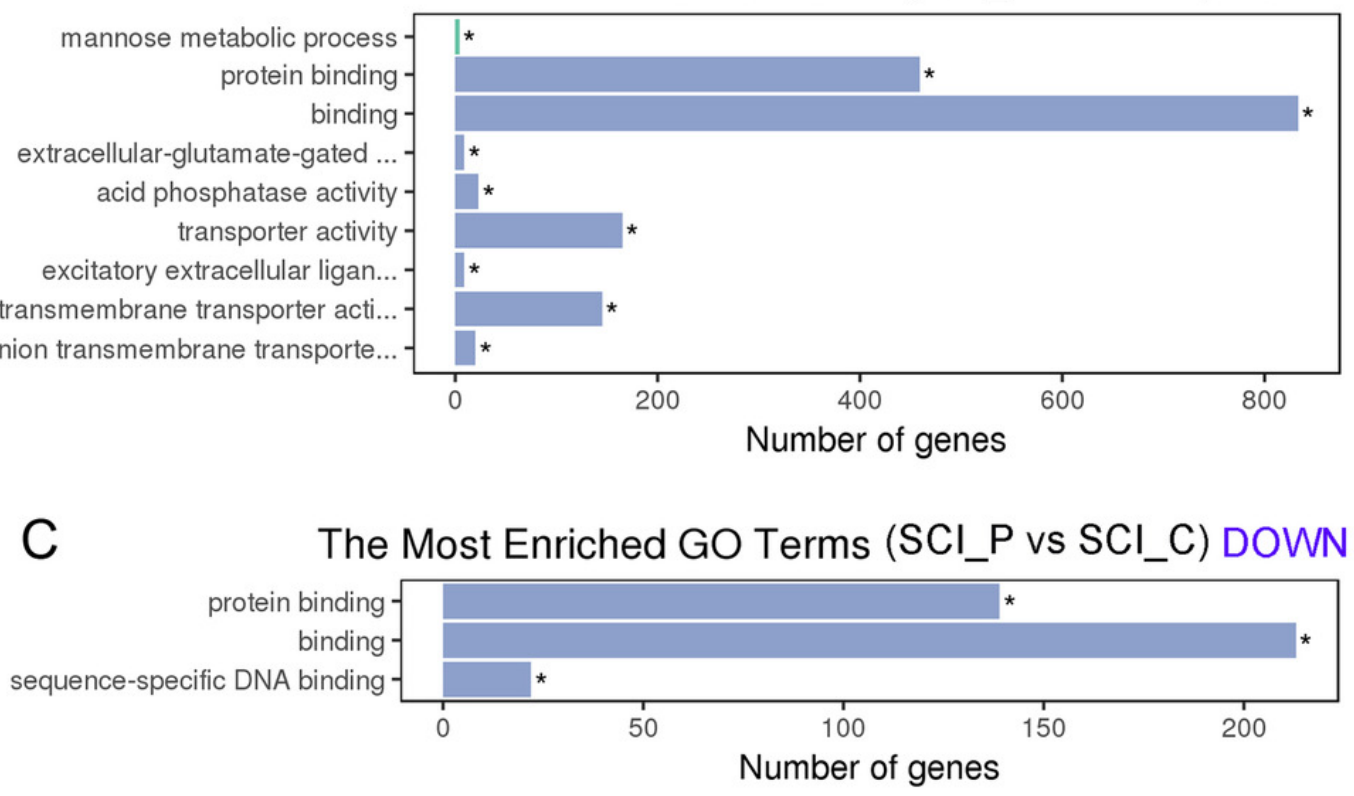

type 


\section{Figure 7}

Figure 7 KEGG enrichment analyses of DEGs

KOBAS software was used to test the statistical enrichment of DEGs in KEGG pathways. In this figure, KEGG enrichment is measured by Rich factor, Qvalue and the number of genes enriched in the related pathway. Rich factor refers to the ratio of the number of differentiated genes (sample number) enriched in the pathway to the number of annotated genes (background number). The larger the Rich factor, the greater the degree of enrichment. Qvalue is the Pvalue after multiple hypothesis test correction. The range of Qvalue is between 0 and 1 . The closer the Qvalue is to 0 , the more significant the enrichment is. The KEGG pathways were shown in A: upregulated DEGs (SCl_C vs sham); B: downregulated DEGs; C: upregulated DEGs (SCl_P vs SCP_C); D: downregulated DEGs (SCl_C vs sham). 


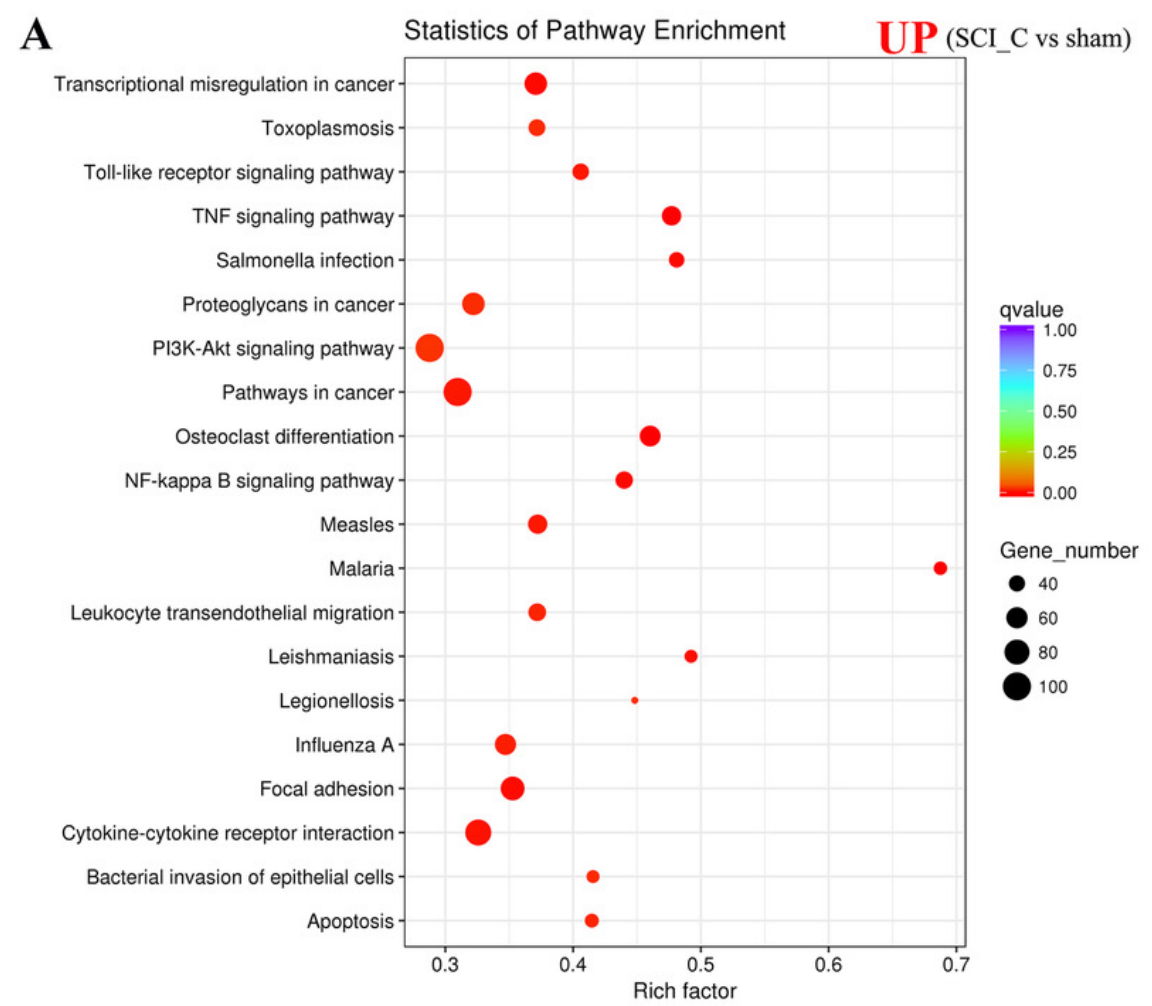

B

Statistics of Pathway Enrichment DOWN (SCI_C vs sham)

qvalue
1.00
0.75
0.50
0.25
0.00

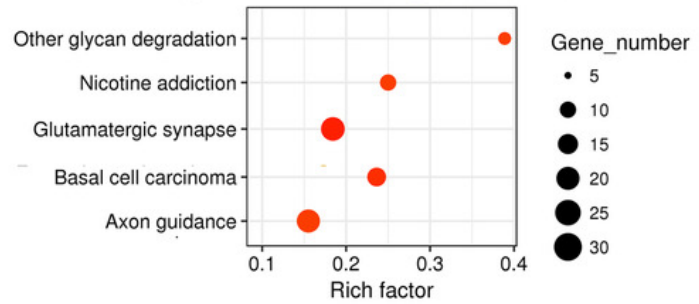

C Statistics of Pathway Enrichment UP (SCI_P vs SCI_C)

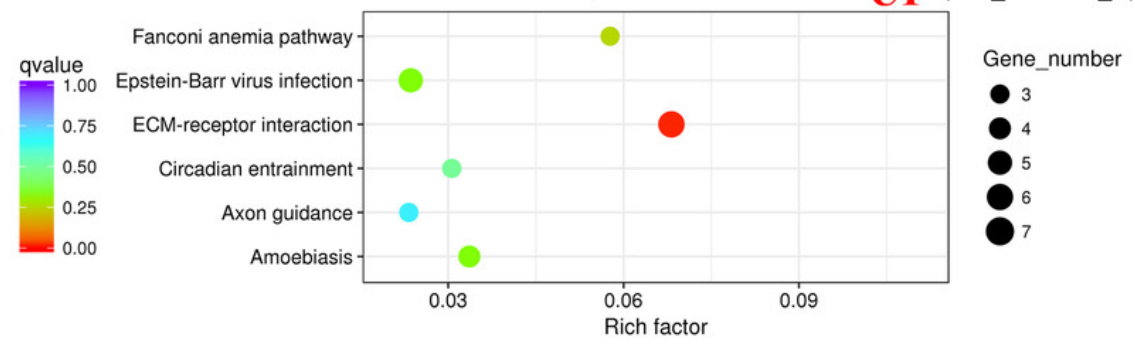

$\mathrm{D}$ Statistics of Pathway Enrichment DOWN(SCI_P vs SCI_C)

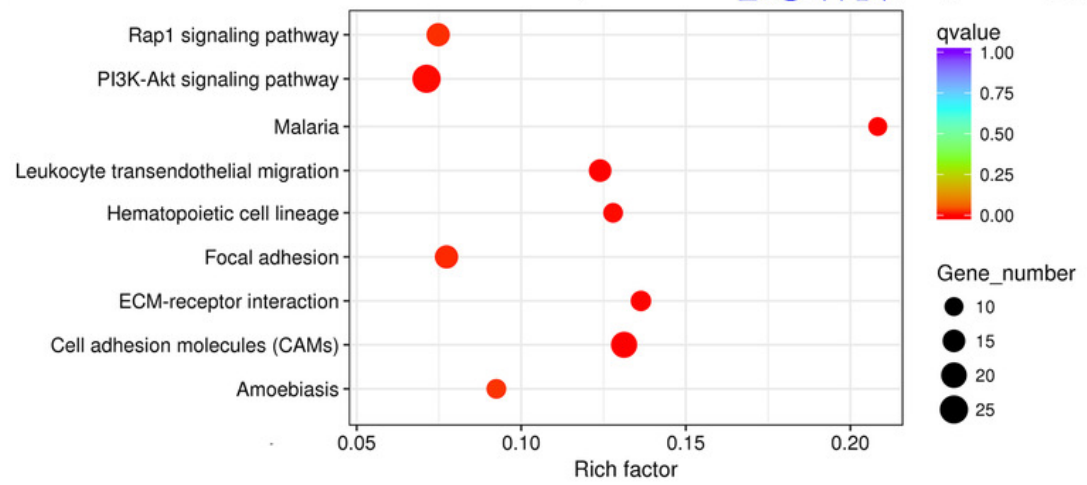

Peer) reviewing PDF | (2019:07:39856:2:0:NEW 25 Nov 2019) 


\section{Table $\mathbf{1}$ (on next page)}

Table 1 PCR primersused in the study 
1 Table 1 Real-time PCR primers used in the study

\begin{tabular}{|c|c|c|}
\hline$\overline{\text { Gene }}$ & Forward primer 5' - 3' & Reverse primer 5' - 3' \\
\hline Itga1 & TCAGTGGAGAGCAGATCGGA & CCTCGTCTGATTCACAGCGT \\
\hline lamb1 & TGCCTTTTCTCCCCGCTACC & CCATGTCCAGTCCTCGCAGA \\
\hline Cldn5 & TTCTATGATCCGACGGTGCC & CTTGACCGGGAAGCTGAACT \\
\hline CD34 & ACCACAGACTTCCCCAACTG & CATATGGCTCGGTGGGTGAT \\
\hline lama2 & GCATTAGTGAGCCGCCCTAT & TCTTTCAGGTCTCGTGTGGC \\
\hline Esam & AGACTCTGGGACTTACCGCT & GGTCACATTGGTCCCGACAT \\
\hline Setdb2 & CCACAAATGGAGATCATACACCT & GCAGTGGGGCTTCCTTTTTC \\
\hline Agrn & CTCTGCCACTGGAACACAGA & GGAAAAGCAGCACCGCAAAG \\
\hline Ccnt2 & AGCAAGGATTTGGCACAGAC & CTCTAGGGTAACCGTGGGGT \\
\hline beta-actin & AGAAGCTGTGCTATGTTGCTCTA & ACCCAAGAAGGAAGGCTGGAAAA \\
\hline
\end{tabular}




\section{Table 2 (on next page)}

Table 2 Summary of sequence assembly after Illumina sequencing

Sham: Sham_1, Sham_2, Sham_3; SCl (solvent control): SCl_C1, SCI_C2, SCI_C3; SCl

(probenecid): SCl_P1, SCl_P2, SCl_P3; Q20: The percentage of bases with a Phred value > 20; Q30: The percentage of bases with a Phred value $>30$. 
1 Table 2 Summary of sequence assembly after Illumina sequencing

2

\begin{tabular}{llllllll}
\hline $\begin{array}{l}\text { Sample } \\
\text { name }\end{array}$ & Raw reads & Clean reads & $\begin{array}{l}\text { clean } \\
\text { bases }\end{array}$ & $\begin{array}{l}\text { Error } \\
\text { rate }(\%)\end{array}$ & $\begin{array}{l}\text { Q20 } \\
(\%)\end{array}$ & $\begin{array}{l}\text { Q30 } \\
(\%)\end{array}$ & $\begin{array}{l}\text { GC content } \\
(\%)\end{array}$ \\
\hline Sham_1 & 56509230 & 55796658 & $8.37 \mathrm{G}$ & 0.03 & 97.73 & 93.95 & 51.23 \\
Sham_2 & 48848744 & 48226002 & $7.23 \mathrm{G}$ & 0.03 & 97.6 & 93.67 & 51.71 \\
Sham_3 & 58228350 & 57459748 & $8.62 \mathrm{G}$ & 0.03 & 97.67 & 93.78 & 51.42 \\
SCI_C1 & 58862872 & 58126844 & $8.72 \mathrm{G}$ & 0.03 & 97.88 & 94.31 & 51.39 \\
SCI_C2 & 56980070 & 56166058 & $8.42 \mathrm{G}$ & 0.03 & 97.74 & 94.03 & 51.42 \\
SCI_C3 & 59804518 & 58798224 & $8.82 \mathrm{G}$ & 0.03 & 97.63 & 93.74 & 51.02 \\
SCI_P1 & 54853344 & 53996254 & $8.1 \mathrm{G}$ & 0.03 & 97.72 & 93.91 & 50.93 \\
SCI_P2 & 56322736 & 55540308 & $8.33 \mathrm{G}$ & 0.03 & 97.87 & 94.27 & 50.94 \\
SCI_P3 & 61037096 & 60037772 & $9.01 \mathrm{G}$ & 0.03 & 97.71 & 93.89 & 50.92 \\
\hline
\end{tabular}

3 Sham: Sham_1, Sham_2, Sham_3; SCI (solvent control): SCI_C1, SCI_C2, SCI_C3; SCI (probenecid): SCI_P1, SCI_P2,

4 SCI_P3;

5 Q20: The percentage of bases with a Phred value $>20$;

6 Q30: The percentage of bases with a Phred value $>30$.

7 